\title{
FEC Performance in Multimedia Streaming
}

\author{
Pascal Frossard
}

\begin{abstract}
In this letter, the performance of packet-level media-independent forward error correction (FEC) schemes are computed in terms of both packet loss ratio and average burst length of multimedia data after error recovery. The set of equations leading to the analytical formulation of both parameters are first given for a renewal error process. Finally, the FEC performance parameters are computed for a Gilbert-model loss process and compared to various experimental data.
\end{abstract}

Index Terms-ABL, forward-error correction, Gilbert model, multimedia streaming, PLR.

\section{INTRODUCTION}

$\mathbf{F}$ ORWARD-ERROR correction (FEC) techniques are the preferred error-control schemes for multicast or interactive streaming applications. In this case, packet-level FEC schemes [1], [2] provide an efficient way to fight against losses, although the perfect recovery cannot be guaranteed.

Several studies have been performed to compute the FEC efficiency or the probability for data to be recovered in case of loss [3]-[5]. However, this parameter does not bring enough information about the loss process in multimedia streaming applications, and more particularly for video and audio streams. To correctly model the video quality, for example, at least two parameters, namely the packet loss ratio $\pi_{v}$ (i.e., the proportion of lost packets) and the average burst length $\alpha_{v}$, shall be computed [6]. The aim of this paper is to compute these two parameters in the case of a renewal error process. The FEC efficiency depends on both the network performance and the media-independent FEC parameters $k$ and $n$.

The paper is organized as follows: Section II presents a brief overview of FEC error-control and develops the set of equations needed to compute $\pi_{v}$ and $\alpha_{v}$. Both parameters are then computed in Section III for a Gilbert-model loss process. The analytical values are validated against experimental data. Finally, concluding remarks are given in Section IV.

\section{LOSS PROCESS AFter FEC RECOVERY}

Recall that common FEC schemes based on Reed-Solomon codes or X-OR functions can generally correct as many losses as the number of redundancy packets. In media-independent FEC,

\footnotetext{
Manuscript received August 21, 2000. The associate editor coordinating the review of this paper and approving it for publication was Prof. A. Haimovich. This work was supported by Hewlett Packard.

The author is with the Signal Processing Laboratory, Swiss Federal Institute of Technology, CH-1015 Lausanne, Switzerland (e-mail: pascal.frossard@epfl.ch).

Publisher Item Identifier S 1089-7798(01)02809-5.
}

every block of $k$ video ${ }^{1}$ packets are followed by $(n-k)$ FEC packets to form a $n$-packet FEC block. If at least $k$ out of $n$ packets are correctly received, the underlying video information can be correctly decoded. Otherwise, none of the lost packets can be recovered by the receiver.

Let us assume that the loss process can be modeled with a renewal error process. In other words, the lengths of consecutive inter-error intervals (also called gaps) are assumed to be independently and identically distributed. For the sake of clarity assume any packet is assigned a binary value 0 or 1 corresponding to correctly received and lost packets respectively.

Following the development of [3], let $p(i)$ denote the probability that a gap length is $i-1$, i.e., $p(i)=\operatorname{Pr}\left(0^{i-1} 1 \mid 1\right)$, where $0^{i-1}$ is a shorthand for $i-1$ successive 0 's. Similarly, let $P(i)$ denote the probability that at least $i-10$ 's follow a given error, i.e., $P(i)=\operatorname{Pr}\left(0^{i-1} \mid 1\right)$.

Order is irrelevant because of the independence among gap lengths of a renewal process. The events $10^{i-1}$ and $0^{i-1} 1$ are therefore equiprobable. From this property, the probability $R(m, n)$ that $m-1$ errors occur in the next $n-1$ bits following an error can be easily computed by recurrence [3]. Thus,

$$
R(m, n)= \begin{cases}P(n), & \text { for } m=1 \text { and } n \geq 1 \\ \sum_{i=1}^{n-m+1} p(i) R(m-1, & n-i), \\ & \text { for } 2 \leq m \leq n .\end{cases}
$$

Similarly, we denote $r(m, n)$ the probability that $m-1$ errors occur in the $n-1$ bits between two errors. It can also be computed by recurrence as

$r(m, n)= \begin{cases}p(n), & \text { for } m=1 \text { and } n \geq 1 \\ \sum_{i=1}^{n-m+1} p(i) r(m-1, & n-i), \\ & \text { for } 2 \leq m \leq n\end{cases}$

Finally, let $\bar{r}(m, n)$ represent the probability that $m-1$ errors occur in the $n-1$ following an error and preceding a 0 :

$$
\bar{r}(m, n)=R(m, n)-r(m, n) .
$$

Let moreover define the probability $q(i)$ that a burst is of length $i-1$ and the probability $Q(i)$ that at least $i-11$ 's follow a zero. These probabilities are given by the loss process or can even be deduced from the above variables. The dual of $R(m, n)$, namely $S(m, n)$, represents the probability to have $m-10$ 's

\footnotetext{
${ }^{1}$ This study is completely valid for any other multimedia stream, as long as the FEC schemes remains a appropriate protection mechanism.
} 
in the next $n-1$ bits following a 0 . This probability is obtained by recurrence from

$S(m, n)= \begin{cases}Q(n), & \text { for } m=1 \text { and } n \geq 1 \\ \sum_{i=1}^{n-m+1} q(i) S(m-1, n-i), & \text { for } 2 \leq m \leq n\end{cases}$

The video packet loss rate after FEC recovery is now easy to compute. Two cases are considered with respect to the state of the last video packet of a FEC block. Its loss or its presence directly drives the loss process into the next FEC block. By the renewal process properties, $\pi_{v}$ could thus be computed as

$$
\begin{aligned}
\pi_{v}= & \frac{\pi}{k} \sum_{i=1}^{k} i R(i, k) \sum_{j=\lfloor n-k+1-i\rfloor}^{n-k} R(j+1, n-k+1) \\
& +\frac{1-\pi}{k} \sum_{i=1}^{k-1}(k-i) S(i, k) \sum_{j=0}^{k-1-i} S(j+1, n-k+1)
\end{aligned}
$$

where the notation $\lfloor x\rfloor$ represents the positive part of $x$ and $\pi$ represents the global packet loss ratio.

The average video burst length after FEC recovery, $\alpha_{v}$, can also be computed from the previous development. Since bursts of errors do not have the same probability to start on any packet of the FEC block, each position has first to be considered separately. The probability for a burst to start on the $j$ th packet of a FEC block (i.e., $1 \leq j \leq n$ ) is given by

$$
P_{j}= \begin{cases}\pi\left(P(2)+p(1) R_{n}\right) \sum_{i=n-k}^{n-1} R(i+1, n), & \text { if } j=1 \\ \sum_{i=0}^{n-j} \sum_{x=0}^{k-n+j+i-2} S(x+1, j-1) & \text { if } j>1\end{cases}
$$

where $R_{n}$ is the probability that all packets in a FEC block are recovered given that the first packet is missing. It can be written as

$$
R_{n}=\sum_{i=0}^{n-k-1} R(i+1, n)
$$

The average length of bursts of lost video packets, $\alpha_{v}$, excludes redundancy packets. Let first $T$ denote the number of FEC blocks transitions along the burst of length $l$ :

$$
T=\text { floor }\left(\frac{l+j-2}{k}\right) \text {. }
$$

Let $v$ denote the position in a FEC block of the first video packet following a burst of length $l$ :

$$
v= \begin{cases}k, & \text { if }(l+j-1) \bmod k=0 \\ (l+j-1) \bmod k, & \text { otherwise. }\end{cases}
$$

Moreover, let $r_{n-k}$ and $\bar{r}_{n-k}$ denote the probability to loose all the video packets of a FEC block and that the first video packet of the next FEC block is, respectively, erased or not (before FEC recovery). These probabilities can be written as

$$
\begin{aligned}
& r_{n-k}=\sum_{\lfloor n-2 k+1\rfloor}^{n-k} r(i+1, n-k+1) \\
& \bar{r}_{n-k}=\sum_{\lfloor n-2 k+1\rfloor}^{n-k} \bar{r}(i+1, n-k+1) .
\end{aligned}
$$

Similarly $r_{n-k}^{j}$ and $\bar{r}_{n-k}^{j}$ denote the probability that the last video packet (i.e., the packet $k$ ) of the first FEC block is lost and the first video packet of the second block is respectively erased or not (before FEC recovery). It is assumed that all video packets between packets $j$ and $k$ are lost. These probabilities are expressed as

$$
\begin{aligned}
r_{n-k}^{j} & =\sum_{i=0}^{n-k} r(i+1, n-k+1) \sum_{x=0}^{2 k-n+i-2} S(x+1, j-1) \\
\bar{r}_{n-k}^{j} & =\sum_{i=0}^{n-k} \bar{r}(i+1, n-k+1) \sum_{x=0}^{2 k-n+i-2} S(x+1, j-1) .
\end{aligned}
$$

Finally, the average length of a burst of lost video packets starting on the $j$ th video packet of a FEC block is given by

$$
L_{j}=\sum_{l=0}^{\infty} l p(1)^{l-T-1} \lambda_{j}(l)
$$

where $\lambda_{j}(l)$ is the probability to have a video packet burst of length $l$ starting at $j$. The conditional probability $\lambda_{1}(l)$ for bursts starting with the first video packet of the FEC block (i.e., $j=1$ ) can be written as

$$
\lambda_{1}(l)=\left\{\begin{array}{lc}
\frac{\pi\left(P(2)+p(1) R_{n}\right)}{P_{1}}\left(r_{n-k} R_{n}+\bar{r}_{n-k}\right) r_{n-k}{ }^{T}, \\
\frac{\pi\left(P(2)+p(1) R_{n}\right)}{P_{1}} P(2) & \text { if } v=n \\
\cdot \sum_{i=0}^{k-2} S(i+1, n-v) r_{n-k}{ }^{T}, & \text { otherwise. }
\end{array}\right.
$$

For $2 \leq j \leq k, \lambda_{j}$ can be written as

$$
\begin{aligned}
& \lambda_{j}(l)= \\
& \left\{\begin{array}{l}
\frac{\pi p(1)}{P_{j}} \sum_{i=0}^{n-v-1} \sum_{x=0}^{k-3-i} S(x+1, j-1) p(1) S(i+1, n-v), \\
\frac{\pi p(1)}{P_{j}}\left(r_{n-k}^{j} l+j-1<k,\right. \\
\frac{\pi p(1)}{P_{j}}\left(r_{n-k} R_{n} R_{n-k}+\bar{r}_{n-k}\right), \quad \text { if } v=k \text { and } T=0 \\
\frac{\pi p(1)}{P_{j}} p(1) \sum_{i=0}^{k-2} S(i+1, n-v) r_{n-k}^{j} r_{n-k} r^{T}, \quad \text { if } v=k \text { and } T \neq 0
\end{array}\right.
\end{aligned}
$$

Finally, $\alpha_{v}$ is given from (6) and (7) by

$$
\alpha_{v}=\left(\sum_{j=1}^{k} P_{j} L_{j}\right) / \sum_{j=1}^{k} P_{j} .
$$




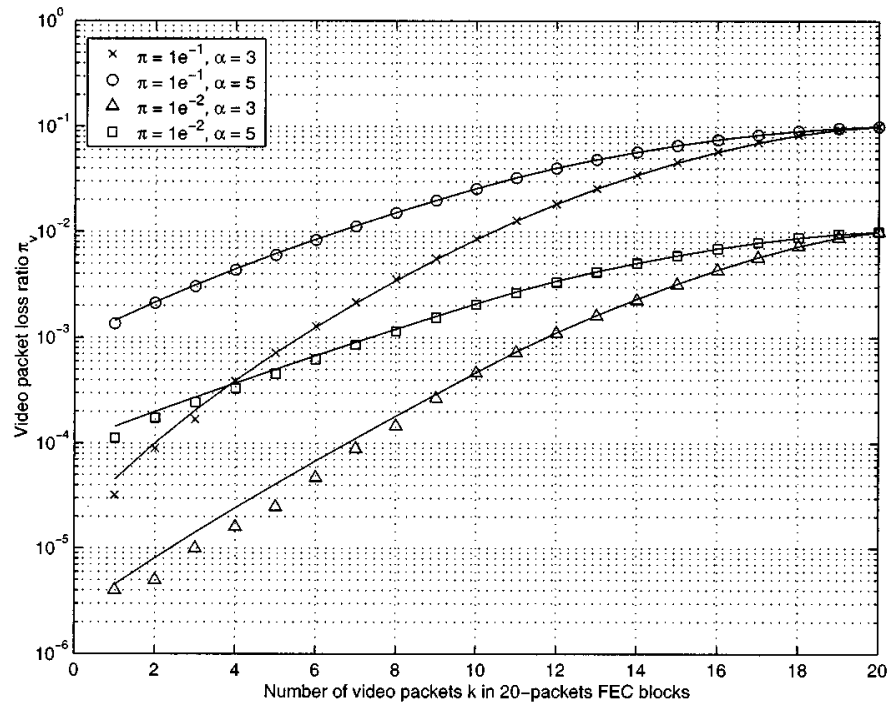

Fig. 1. Evolution of $\pi_{v}$ versus the number of video packets $k$ in a FEC block of length $n=20$ in a Gilbert-model loss process.

\section{FEC IN A GILBert-Model Loss Process}

Assume now that the channel loss process can be characterized by the Gilbert model [7]-[10]. The Gilbert model is a two-state Markovian model [11] with geometrically distributed residence times. States 0 and 1 correspond respectively to the correct reception or the loss of a packet. The transition rates $p$ and $q$ between the states control the lengths of the error bursts.

For a Gilbert loss process the following relations hold:

$$
\begin{aligned}
p(i) & = \begin{cases}1-q, & \text { if } i=1 \\
q(1-p)^{i-2} p, & \text { otherwise }\end{cases} \\
P(i) & = \begin{cases}1, & \text { if } i=1 \\
q(1-p)^{i-2}, & \text { otherwise }\end{cases} \\
q(i) & = \begin{cases}1-p, & \text { if } i=1 \\
p(1-q)^{i-2} q, & \text { otherwise }\end{cases} \\
Q(i) & = \begin{cases}1, & \text { if } i=1 \\
p(1-q)^{i-2}, & \text { otherwise. }\end{cases}
\end{aligned}
$$

The probabilities $R(m, n), r(m, n), \bar{r}(m, n)$ and $S(m, n)$ can be computed by recurrence from (1)-(4) respectively. The video packet loss ratio and average burst length are then computed from (5) and (8), respectively.

Figs. 1 and 2 represent the evolution of the video loss parameters $\pi_{v}$ and $\alpha_{v}$ for different network loss patterns. The analytical values perfectly fit the experimental data. Moreover, $\pi_{v}$ obviously increases with $k$ as the amount of protection decreases for a given $n$. FEC protection becomes also less efficient for bursty loss traffic (i.e., large $\alpha$ values) for a given $\pi$. Moreover the average length of lost video packets clearly exhibits a maximum. This can be explained as follows. When the amount of protection is very large $\alpha_{v}$ stays close to $k$. When the amount of protection decreases, the video loss pattern get closer to the channel loss pattern. In between there is a maximum which is less pronounced for bursty process. These behaviors still hold for different FEC block lengths.

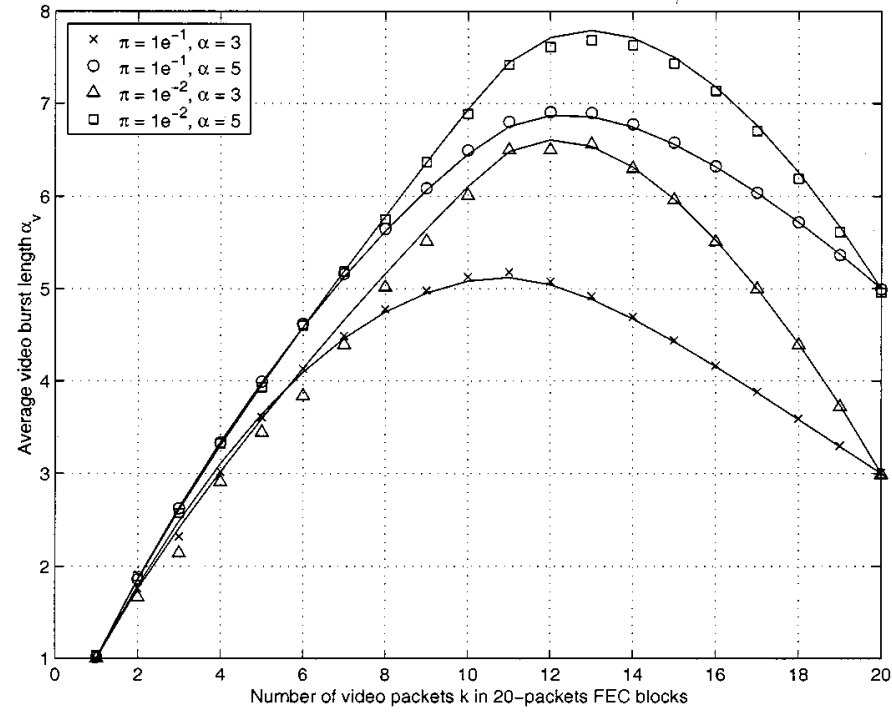

Fig. 2. Evolution of $\alpha_{v}$ versus the number of video packets $k$ in a FEC block of length $n=20$ in a Gilbert-model loss process.

\section{CONCLUSIONS}

In this letter the performance of a media-independent FEC scheme for video streaming applications is analyzed. Both the packet loss ratio and average burst length as perceived by the multimedia application are computed for a Gilbert-model loss process. Experimental simulations validate the analytical results. Our main results can be used to compute the optimal FEC parameters $k$ and $n$ for a given packet loss process as seen by the network. Clearly, the results in this paper hold for any type of multimedia data for which FEC protection is suitable.

\section{REFERENCES}

[1] L. Rizzo, "Effective erasure codes for reliable computer communication protocols," ACM Comput. Commun. Rev., vol. 27, no. 2, pp. 24-36, Apr. 1997.

[2] N. Shacham and P. McKenney, "Packet recovery in high-speed networks using coding and buffer management," in Proc. IEEE INFOCOM Conference, vol. 1, Los Alamitos, CA, 1990, pp. 124-131.

[3] E. O. Elliott, "A model of the switched telephone network for data communications," Bell Syst. Tech. J., vol. 44, no. 1, pp. 89-109, Jan. 1965.

[4] J.-P. A. Adoul, "Error intervals and cluster density in channel modeling," IEEE Trans. Inform. Theory, vol. 20, pp. 125-129, Jan. 1974.

[5] L. N. Kanal and A. R. K. Sastry, "Models for channels with memory and their applications to error control," Proc. IEEE, vol. 66, pp. 724-744, July 1978.

[6] P. Frossard and O. Verscheure, "Joint source/FEC rate selection for optimal MPEG-2 video delivery," in Int. Conf. on Multimedia and Expo, vol. 3, New York, July 2000, pp. 1301-1304.

[7] J. Bolot, S. Fosse-Parisis, and D. Towsley, "Adaptive FEC-based error control for internet telephony," in Proc. IEEE INFOCOM, vol. 3, 1999, pp. 1453-1460.

[8] M. Yajnik, J. Kurose, and D. Towsley, "Packet loss correlation in the MBone Multicast Network: Experimental measurements and Markov chain models," Dep. Computer Science, Univ. of Massachusetts at Amherst, Tech. Rep. 95-115, 1995.

[9] V. Paxson, "End-to-end internet packet dynamics," ACM SIGCOMM Comput.Commun. Rev., vol. 27, no. 4, pp. 139-152, Oct. 1997.

[10] M. C. Jeruchim, P. Balaban, and K. S. Shanmugan, Simulation of Communication Systems. New York: Plenum, 1992.

[11] E. N. Gilbert, "Capacity of a burst-noise channel," Bell Syst. Tech. J., pp. 1253-1265, Sept. 1960. 